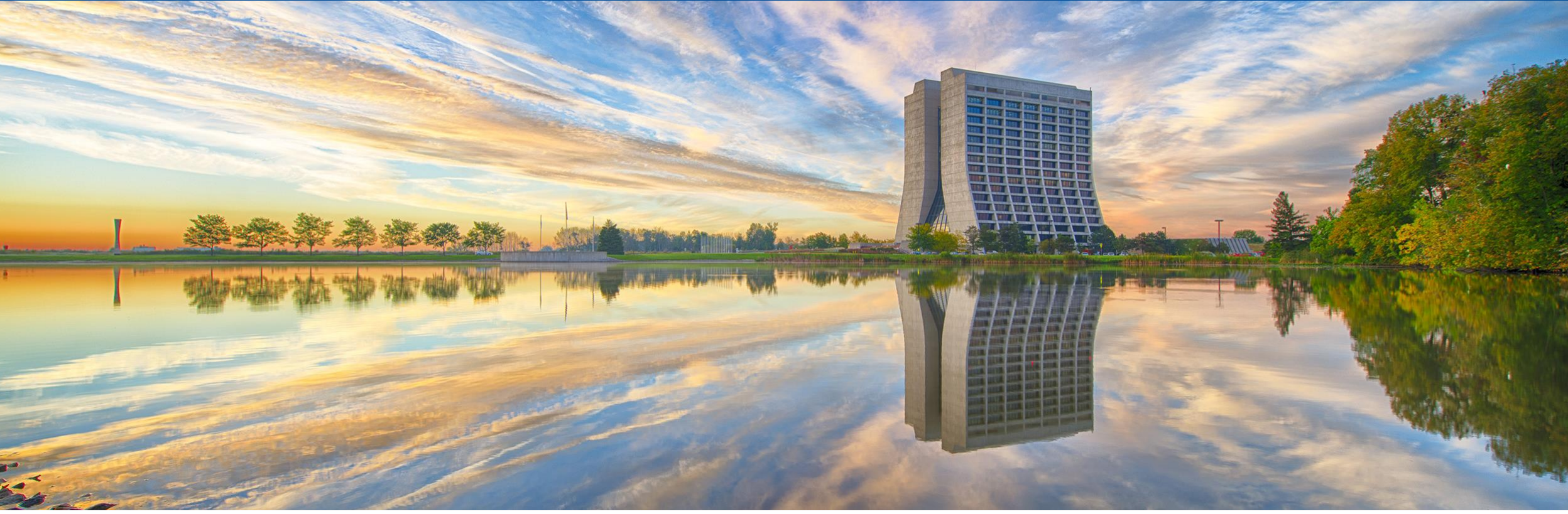

\title{
Study of Fluctuations in Undulator Radiation in the IOTA Ring at Fermilab
}

Ihar Lobach 2 THE UNIVRSITY OF NAPAC2019 Lansing, MI

Thursday Sep $5^{\text {th }}, 2019$
Thesis advisors: Sergei Nagaitsev (UChicago), Giulio Stancari (Fermilab) 


\section{Collaboration}
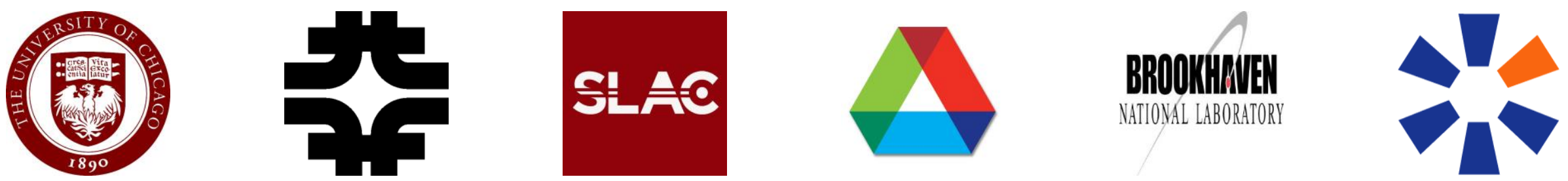

I. Lobach, UChicago, S. Nagaitsev, V. Lebedev, A. Romanov, A. Valishev, G. Stancari, Fermilab, A. Halavanau, Z. Huang, V. Yakimenko, SLAC, A. Murokh, Radiabeam, K. J. Kim, ANL, T. Shaftan, BNL

\section{Acknowledgments:}

We would like to thank the entire FAST/IOTA team for helping us with building and installing the setup, and taking measurements, especially Wayne Johnson, Mark Obrycki, and James Santucci; Greg Saewert, for constructing the photodetector circuit and providing the test light source; David Johnson and Todd Johnson, for kindly providing test equipment and assisting during tests of our detector. Numerous pieces of advice given by Daniil Frolov are greatly appreciated as well. A.H. is grateful to G. Stupakov and Y. Cai (SLAC) for many in-depth physics discussions on the subject. 


\section{Integrable Optics Test Accelerator (IOTA)}

- First beam Aug 21, 2018

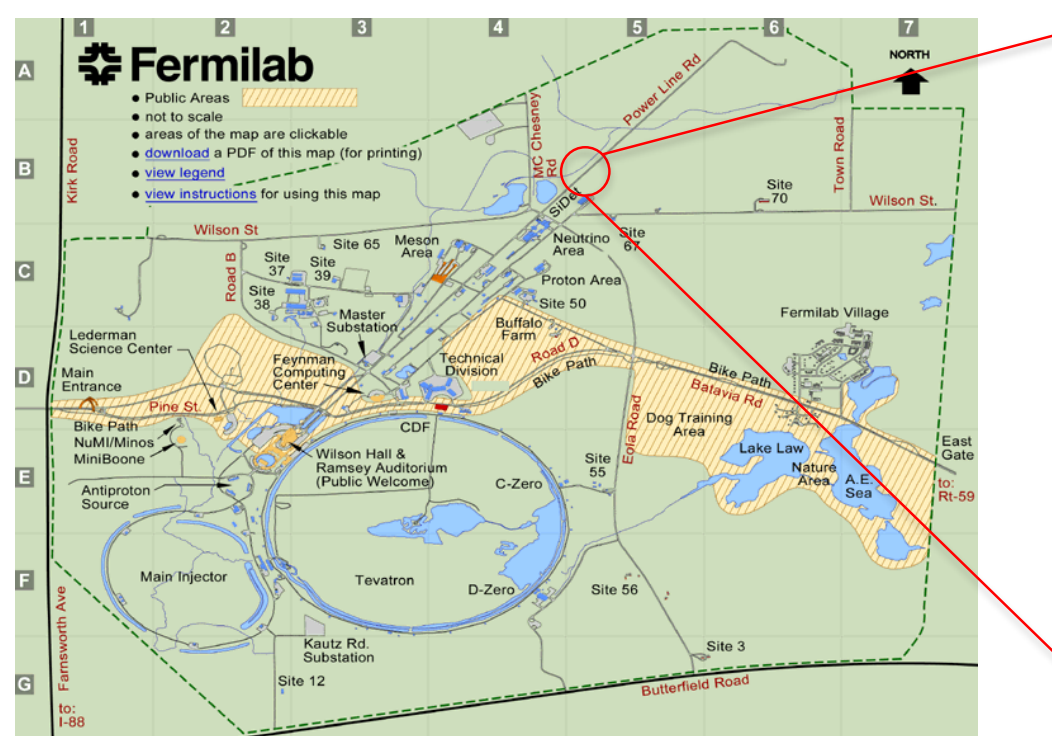

- Particles: electrons/protons

- Main experiments:

- Nonlinear beam optics

- Optical stochastic cooling

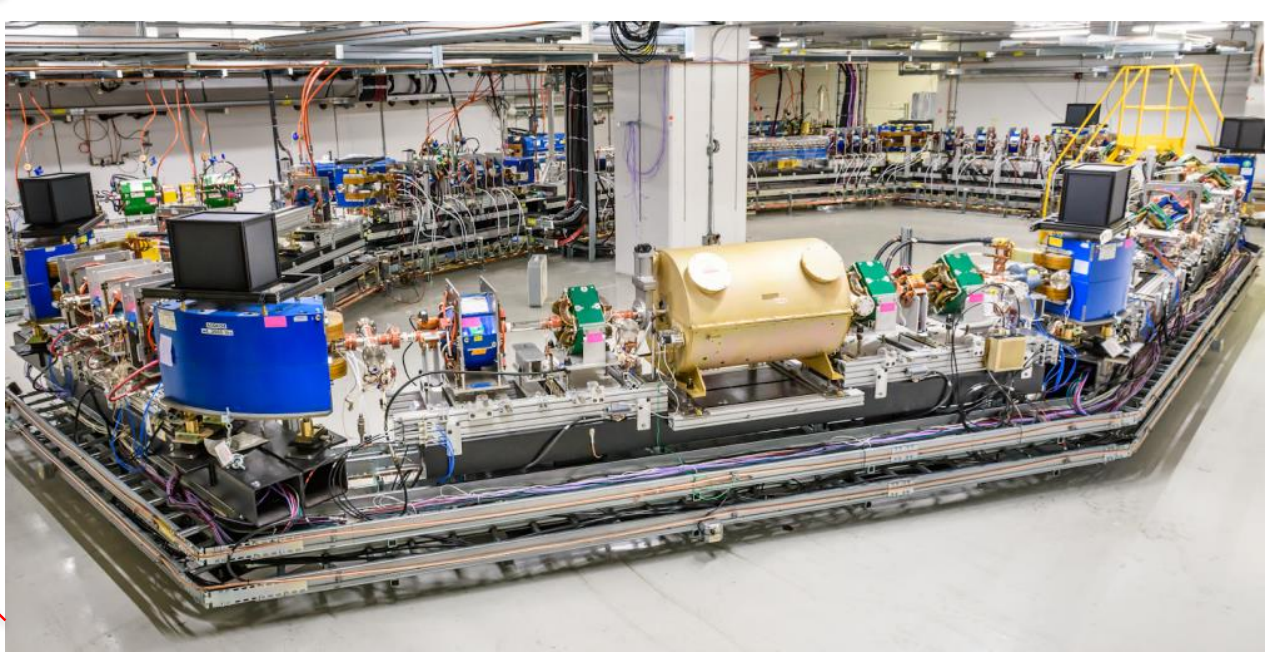

Circumference: $40 \mathrm{~m}$ Electron energy: $100 \mathrm{MeV}$

Not a user facility! 


\section{Experiment idea}
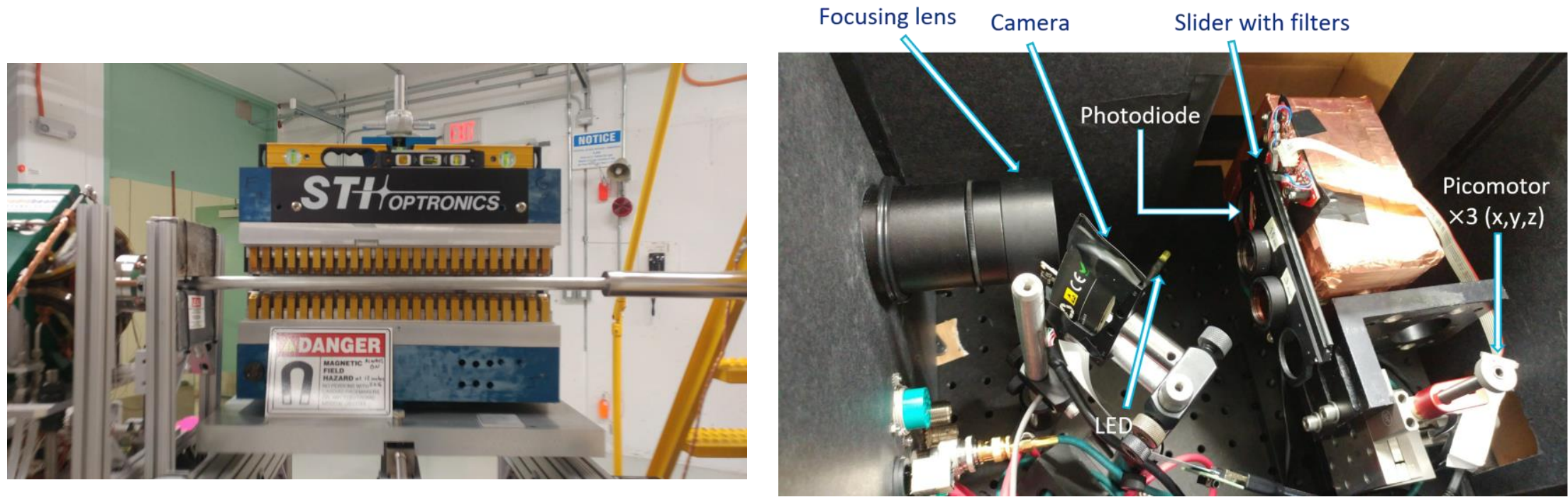

- We installed an undulator in the IOTA ring (late Feb).

- And built an integrating circuit for the photodiode's current. The amplitude of the output voltage was proportional to the number of photoelectrons generated in the photodiode.

- In the experiment (late Mar), we study the fluctuation in the number of photoelectrons, namely, the variance:

$$
\operatorname{var}(\mathcal{N})=\left\langle\mathcal{N}^{2}\right\rangle-\langle\mathcal{N}\rangle^{2}
$$




\section{Theoretical prediction}

Page 28:

Synchrotron

Radiation and

Free-Electron

Lasers

Kwang.je Kim, Zhirong Huang.
and Ryan Linctierg

$$
\operatorname{var}\left(\mathcal{N}_{\mathrm{ph}}\right)=\left\langle\mathcal{N}_{\mathrm{ph}}\right\rangle+\frac{1}{M}\left\langle\mathcal{N}_{\mathrm{ph}}\right\rangle^{2}
$$

Discrete quantum

nature of light
Chaotic light. Incoherent sum over randomly phased electrons

In our experiment:

\#1 Wide band, large solid angle, high QE $=80 \%$

\#2 The two terms are comparable

\#3 RMS fluctuation $\sim 10^{-4}-10^{-3}$

$\frac{d \mathcal{N}_{\mathrm{q} . \mathrm{C} .}^{(1)}}{d \boldsymbol{k}}=\eta_{\boldsymbol{k}} I_{\boldsymbol{k}}^{(1)}$

$\frac{1}{M} \equiv \frac{\operatorname{var}\left(\mathcal{N}_{\text {q.c. }}\right)}{\left\langle\mathcal{N}_{\text {q.c. }}\right\rangle^{2}}=\frac{\frac{\sqrt{\pi}}{\sigma_{z}} \int d k d \Omega_{1} d \Omega_{2} k^{4} \eta_{k n_{1}} I_{k n_{1}}^{(1)} \eta_{k n_{2}} I_{k n_{2}}^{(1)} e^{-k^{2} \sigma_{x}^{2}\left(\theta_{1 x}-\theta_{2 x}\right)^{2}-k^{2} \sigma_{y}^{2}\left(\theta_{1 y}-\theta_{2 y}\right)^{2}}}{\left(\int d \boldsymbol{k} \eta_{\boldsymbol{k}} I_{\boldsymbol{k}}^{(1)}\right)^{2}}$ 


\section{More details on the setup. The photodiode detector}
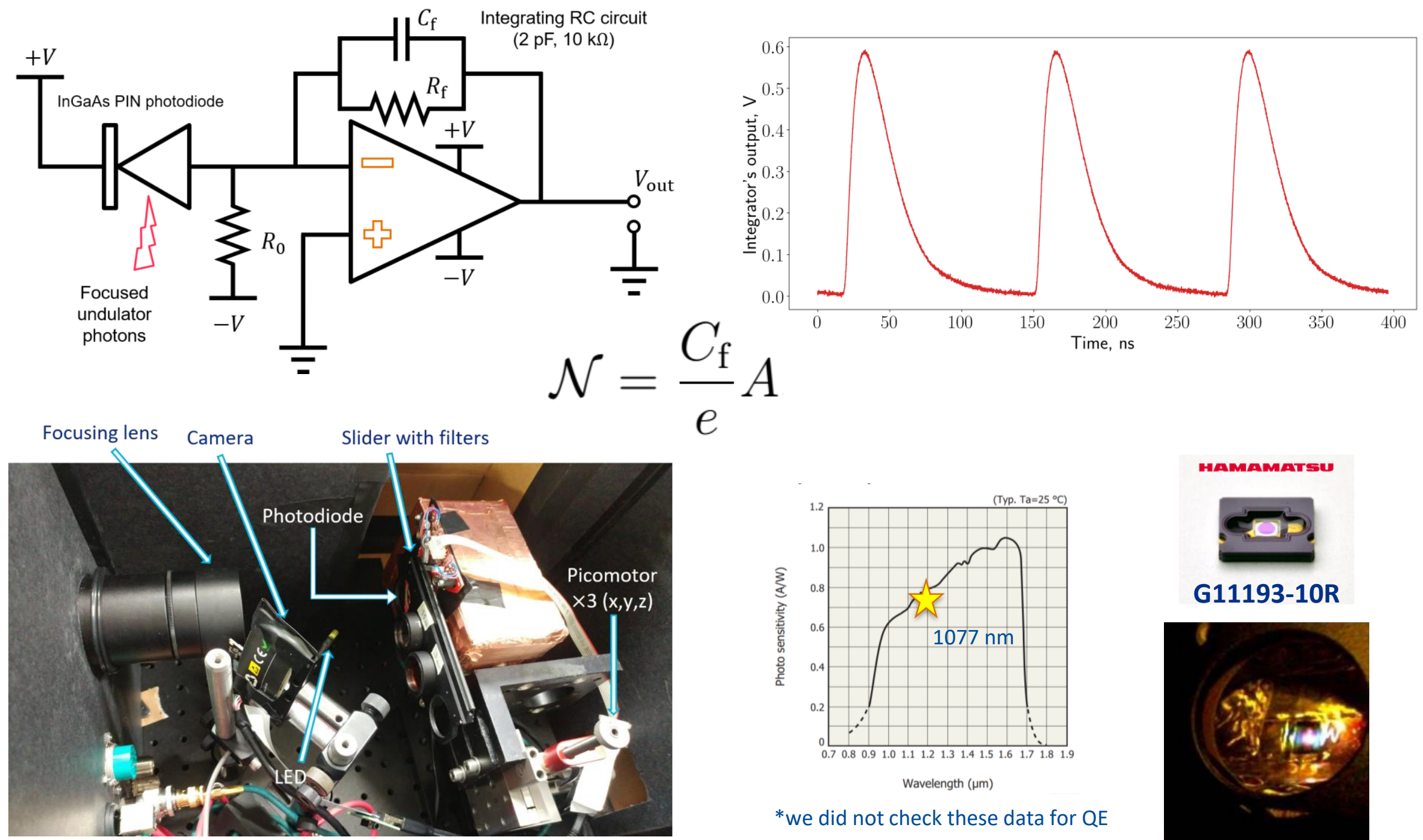

*we did not check these data for QE

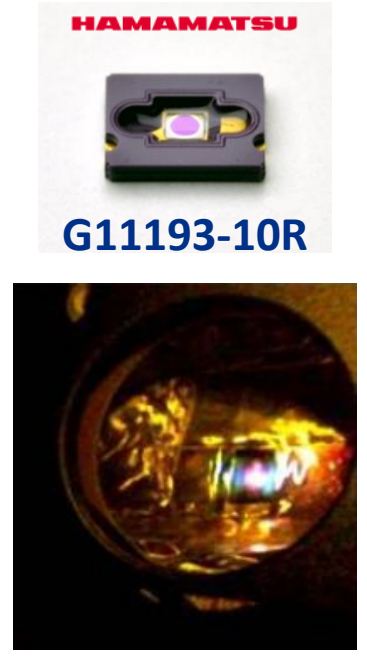




\section{Comb filter. Testing the setup}

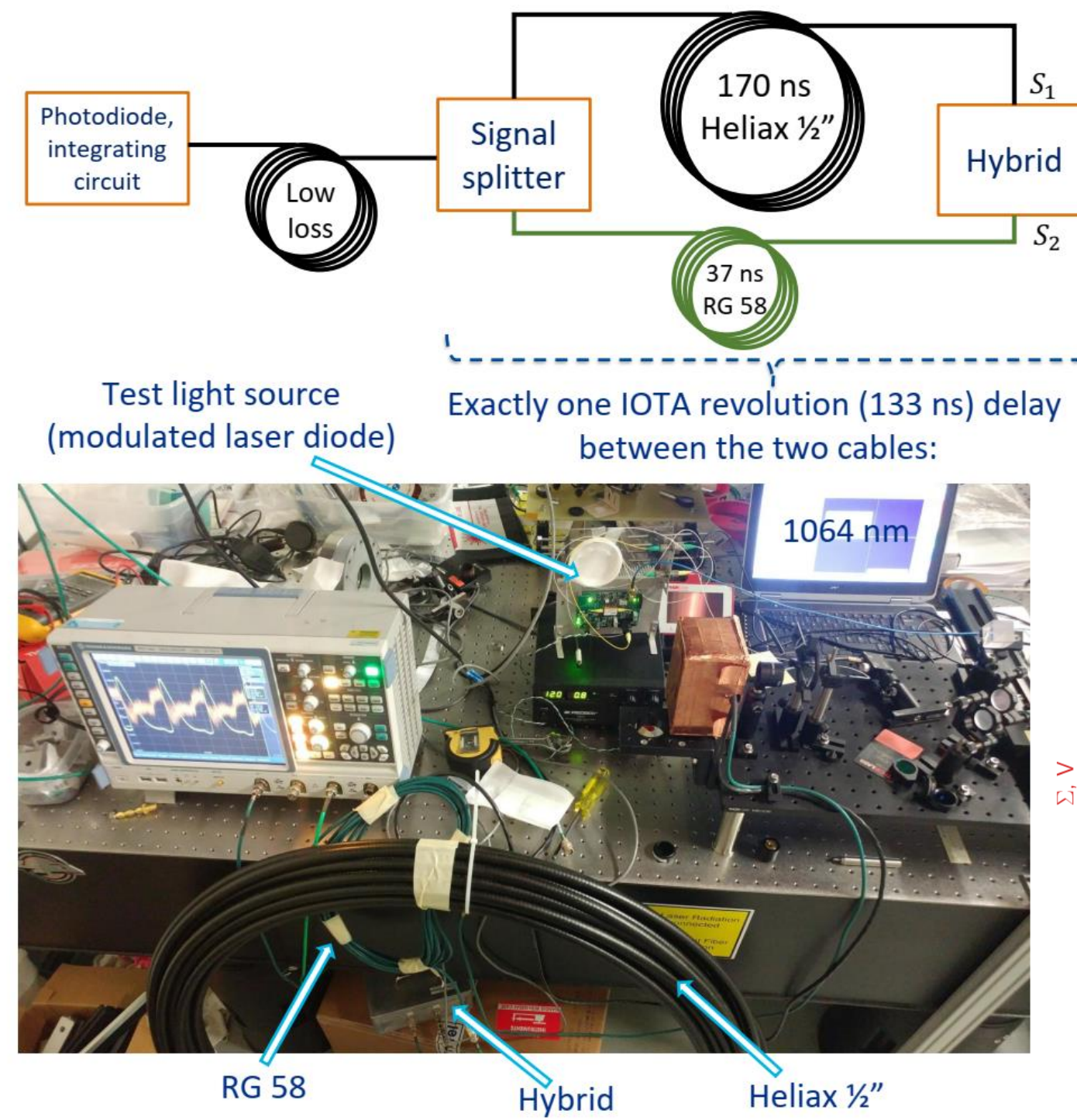

$$
\begin{gathered}
S_{1}=\left(1+\delta_{1}\right) S(t) \\
S_{2}=\left(1+\delta_{2}\right) S(t) \\
\Sigma=S_{1}+S_{2} \approx 2 S(t) \\
\Delta=S_{1}-S_{2}=\left(\delta_{1}-\delta_{2}\right) S(t) \\
A=(1+\delta) S\left(t_{\max }\right) \\
\operatorname{var}\left(\delta_{1}-\delta_{2}\right)=2 \operatorname{var}(\delta) \\
\operatorname{var}(A)=\operatorname{var}\left(\Delta\left(t_{\max }\right)\right) / 2
\end{gathered}
$$

(modulated laser diode) between the two cables:

One dataset is $1.5 \mathrm{~ms}$ ( 11000 turns)

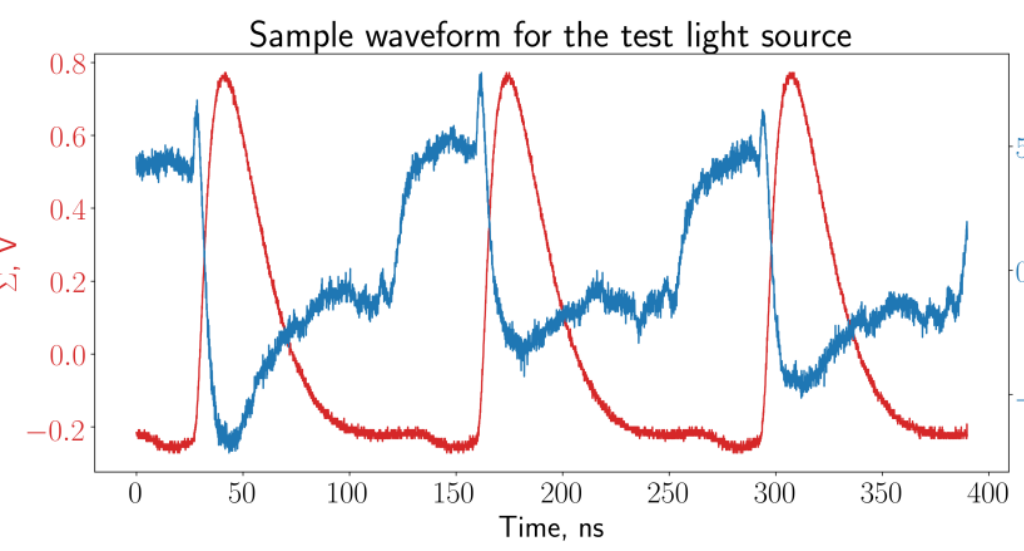

Crosstalk: $0.7 \%$ 


\section{Noise subtraction algorithm}

- In the real experiment with undulator radiation signal/noise $<1$ :

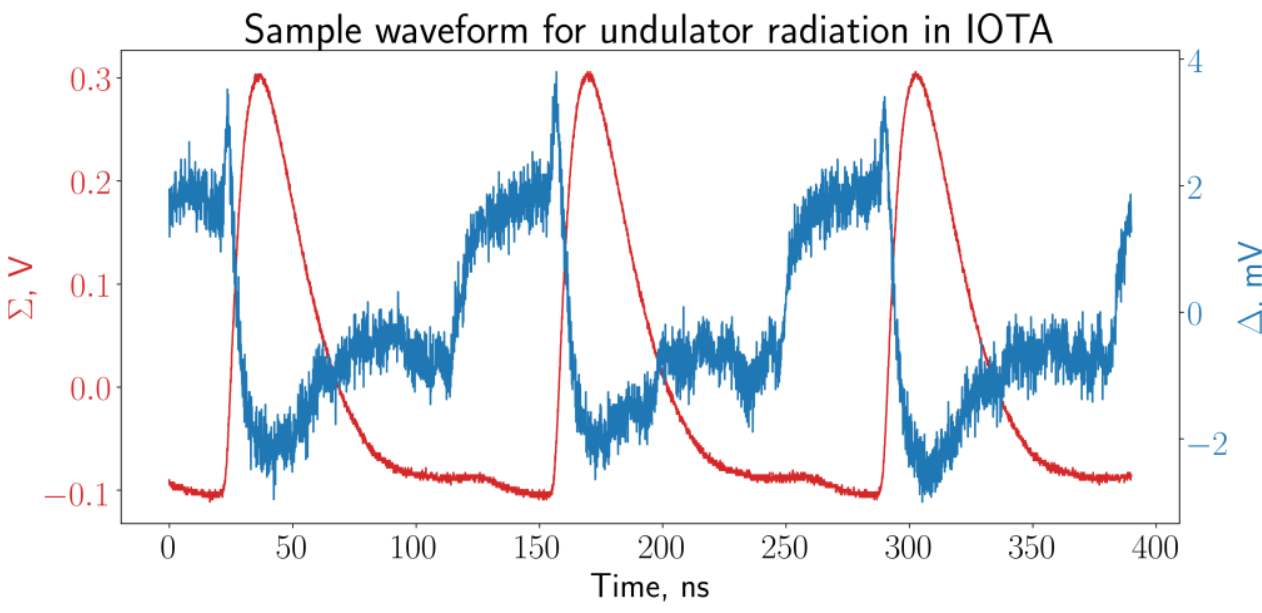

Main sources of noise:

- The oscilloscope

$1 \mathrm{mV}$ peak-to-peak

- The integrator's op-amp

$1.5 \mathrm{mV}$ peak-to-peak (together with the scope)

Total RMS noise: $\approx 0.3 \mathrm{mV}$

Extraction of sub noise level fluctuations:

\#1 Find the period with high accuracy ( $>7$ figures)

\#2 Map all $\Delta$-channel data to one period

\#3 Bin the data along the time axis

\#4 Take variance of $\Delta$-channel in each bin:

$\Delta(t)=\left(\delta_{1}-\delta_{2}\right) S(t)+$ noise

@ fixed t const

$\operatorname{var}(\Delta(t))=2 \operatorname{var}(\delta) S^{2}(t)+\operatorname{var}($ noise $)$

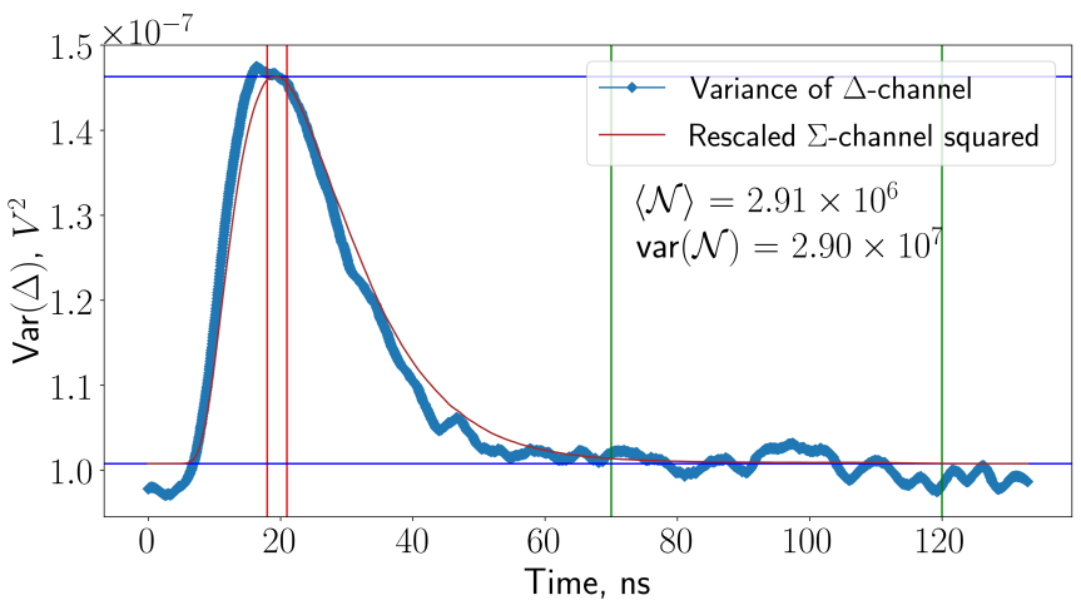




\section{Testing the setup. Finding its precision}

\section{Detector test idea:}

Keep the test light source in the same regime and use different ND filters. Relative classical fluctuation (due to pulse generator and amplifier errors) must stay the same:

$$
\frac{\operatorname{var}(A)}{\langle A\rangle^{2}}=\frac{\operatorname{var}(\mathcal{N})}{\langle\mathcal{N}\rangle^{2}}=\theta=\text { const }
$$

$\theta$ is determined at large $\langle\mathcal{N}\rangle$, when signal/noise $\rangle 1$. Poisson contribution is negligible.
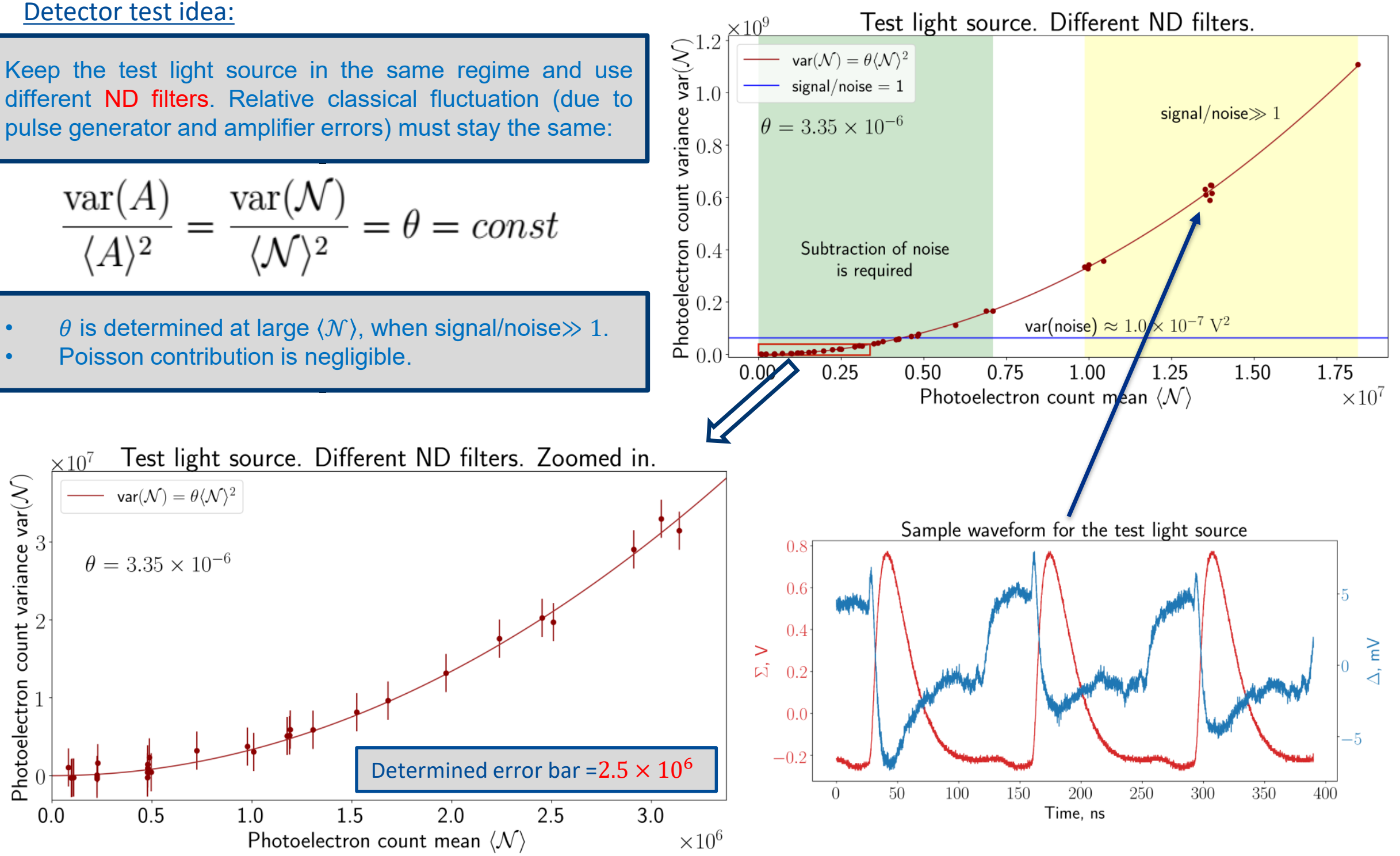

華 Fermilab 


\section{Measurement results in IOTA}

- Constant number of electrons in the bunch, different neutral density (ND) filters in front of the detector
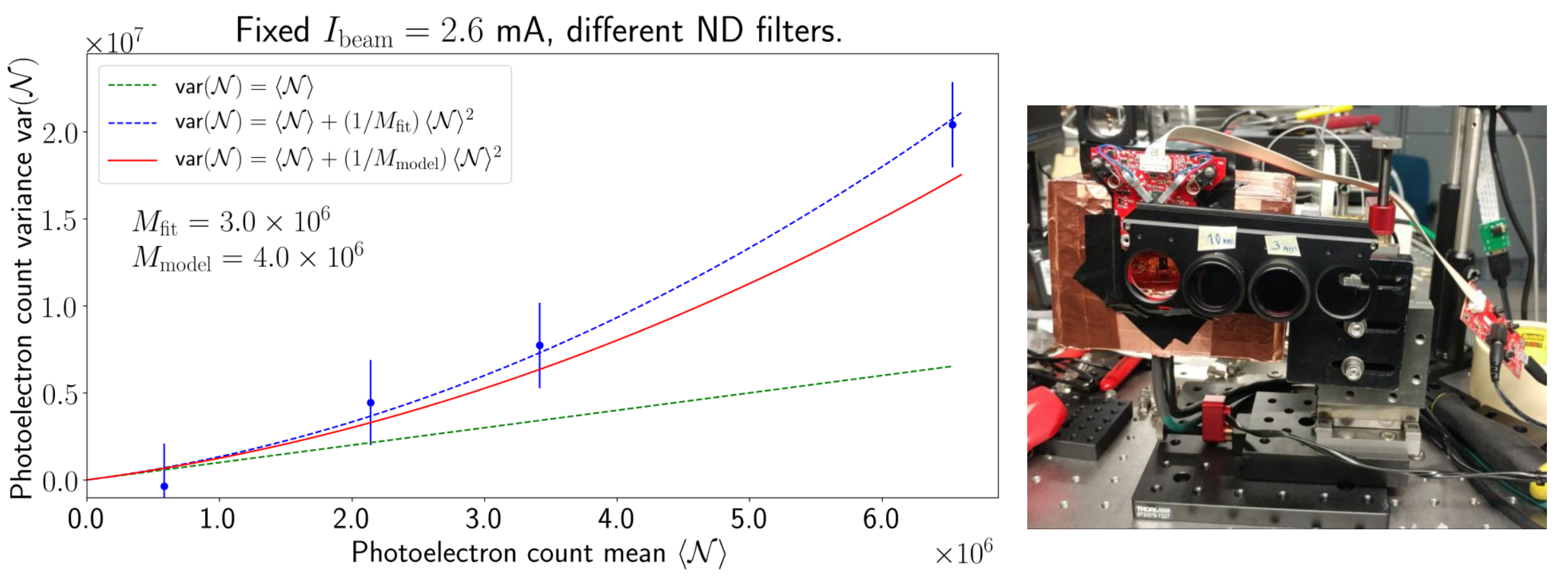

Decreasing optical density of the filters 


\section{Measurement results in IOTA}

- Changing the electron bunch charge. No neutral density (ND) filters in front of the detector.

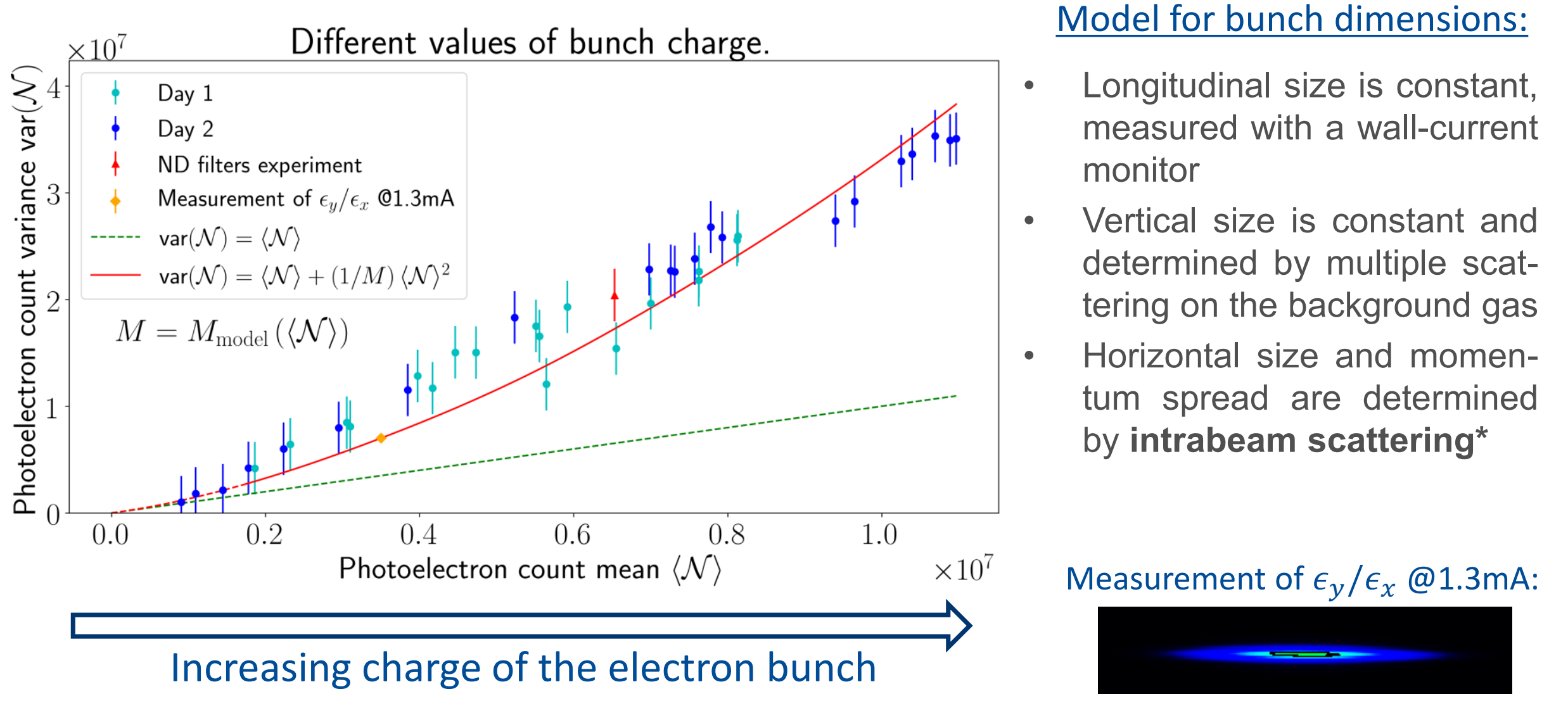

*S. Nagaitsev, Phys. Rev. ST Accel. Beams 8.6 (2005): 064403. 


\section{Conclusions}

- Quantitative theoretical model for the experiment from [1] was developed and verified in an independent experiment in IOTA

- It helped corroborate a model of intrabeam scattering in IOTA. The agreement is expected to improve in the future.

- It was shown that along with measurements of longitudinal bunch size [2-5] the fluctuations can be used to measure transverse bunch size in some cases (e.g., in IOTA).

I. Lobach, V. Lebedev, S. Nagaitsev, A. Romanov, G. Stancari, A. Halavanau, Z. Huang, and K.-J. Kim, Intensity fluctuations in undulator radiation, will be submitted to PRAB.

Improvements as compared to the similar experiment from [1]:

- Better precision due to using the comb filter with one-turn delay and the special noise subtraction algorithm. In IOTA, fluctuations were two orders of magnitude smaller than in [1].

- $\quad$ Fluctuations data were collected for different values of bunch charge.

- The transition from Poisson statistics to Super-Poisson statistics was observed in undulator radiation for the first time.

[1] M. C. Teich, T. Tanabe, T. C. Marshall, and J. Galayda, Statistical properties of wiggler and bending-magnet radiation from the Brookhaven Vacuum-Ultraviolet electron storage ring, Phys. Rev. Lett. 65, 3393 (1990).

[2] F. Sannibale, G. Stupakov, M. Zolotorev, D. Filippetto, and L. Jägerhofer, Absolute bunch length measurements by incoherent radiation fluctuation analysis, Phys. Rev. ST Accel. Beams 12, 032801 (2009).

[3] P. Catravas, W. Leemans, J. Wurtele, M. Zolotorev, M. Babzien, I. Ben-Zvi, Z. Segalov, X.-J. Wang, and
V. Yakimenko, Measurement of electron-beam bunch length and emittance using shot-noise-driven fluctuations in incoherent radiation, Phys. Rev. Lett. 82, 5261 (1999).

[4] V. Sajaev, Measurement of bunch length using spectral analysis of incoherent radiation fluctuations, in AIP Conf. Proc., Vol. 732 (AIP, 2004) pp. 73-87.

[5] V. Sajaev, Determination of longitudinal bunch profile using spectral fluctuations of incoherent radiation, Report No ANL/ASD/CP-100935 (Argonne National Laboratory, 2000). 


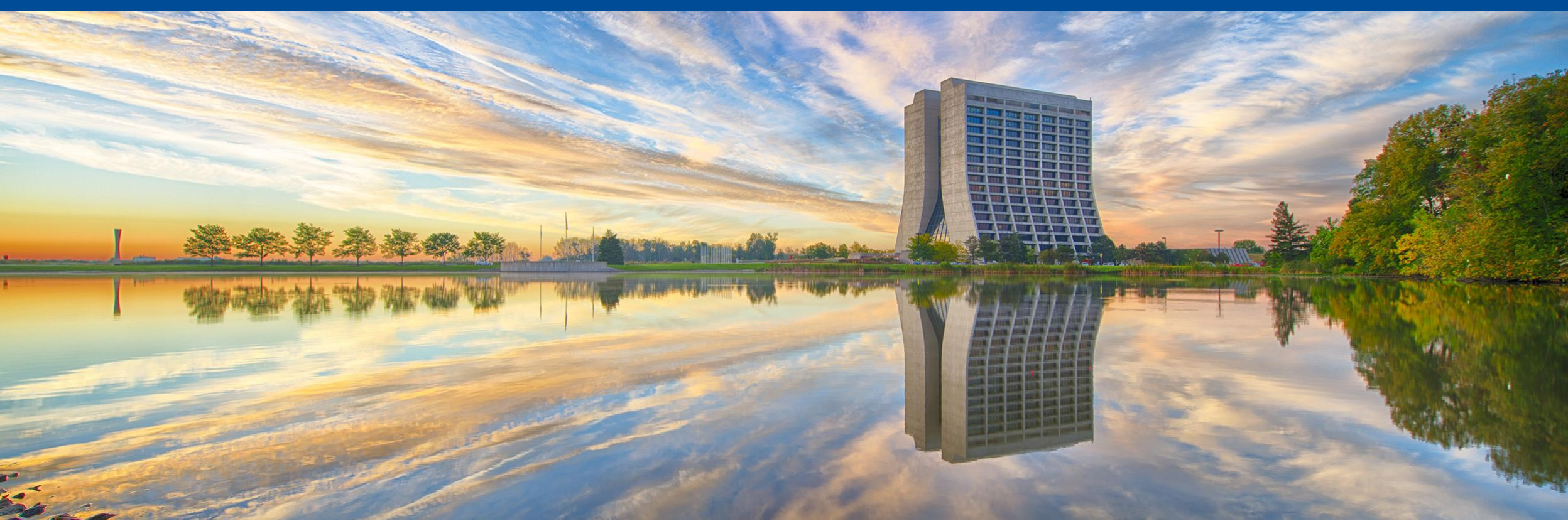

\section{Thank you for your attention!}

